\title{
Overexpression of the Drosophila ATR homologous checkpoint kinase Mei-41 induces a G2/M checkpoint in Drosophila imaginal tissue
}

\author{
Fabienne E. Bayer ${ }^{\dagger}$, Mirjam Zimmermann ${ }^{\dagger}$, Anette Preiss and Anja C. Nagel ${ }^{*}$
}

\begin{abstract}
Background: DNA damage generally results in the activation of ATM/ATR kinases and the downstream checkpoint kinases Chk1/Chk2. In Drosophila melanogaster, the ATR homologue meiotic 41 (mei-41) is pivotal to DNA damage repair and cell cycle checkpoint signalling. Although various mei-41 mutant alleles have been analyzed in the past, no gain-of-function allele is yet available. To fill this gap, we have generated transgenic flies allowing temporal and tissue-specific induction of mei-41.
\end{abstract}

Results: Overexpression of mei-41 in wing and eye anlagen affects proliferation and a G2/M checkpoint even in the absence of genomic stress. Similar consequences were observed following the overexpression of the downstream kinase Grapes (Grp) but not of Loki (Lok), encoding the respective Drosophila Chk1 and Chk2 homologues, in agreement with their previously reported activities. Moreover, we show that irradiation induced cell cycle arrest was prolonged in the presence of ectopic mei-41 expression. Similar to irradiation stress, mei-41 triggered the occurrence of a slower migrating form of Grp, implying specific phosphorylation of Grp in response to either signal. Using a p53R-GFP biosensor, we further show that overexpression of mei-41 was sufficient to elicit a robust p53 activation in vivo.

Conclusion: We conclude that overexpression of the Drosophila ATR homologue mei-41 elicits an effectual DNA damage response irrespective of irradiation.

Keywords: ATR, DNA damage checkpoint, Mei-41, Overexpression, p53 activation

\section{Background}

Environmental and intrinsic stressors may impact the integrity of genome, i.e. the DNA, thereby provoking mutations eventually leading to cellular transformation or cell death. DNA damage is combated by a complex interplay of repair mechanisms ensuring the stability of the genome. Studies on DNA damage response (DDR) in a large variety of organisms, be it single cells like yeast or multicellular organisms like Drosophila or mammals, revealed that all organisms have evolved a core of components strikingly conserved across eukaryotes (reviewed

\footnotetext{
* Correspondence: anja.nagel@uni-hohenheim.de

${ }^{\dagger}$ Fabienne E. Bayer and Mirjam Zimmermann contributed equally to this work.

Universität Hohenheim, Institut für Genetik, Garbenstr. 30, 70599 Stuttgart, Germany
}

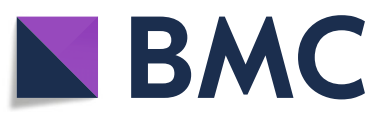

(๑) The Author(s). 2018 Open Access This article is distributed under the terms of the Creative Commons Attribution 4.0 International License (http://creativecommons.org/licenses/by/4.0/), which permits unrestricted use, distribution, and

reproduction in any medium, provided you give appropriate credit to the original author(s) and the source, provide a link to the Creative Commons license, and indicate if changes were made. The Creative Commons Public Domain Dedication waiver (http://creativecommons.org/publicdomain/zero/1.0/) applies to the data made available in this article, unless otherwise stated. ling events, starting with the recognition of DNA lesions followed by the activation of the DNA damage checkpoint pathway to cause a temporarily cell cycle arrest thus enabling DNA repair processes to occur (reviewed in $[1,4])$. Typical of signalling cascades, DDR is regulated by phosphorylation events mediated by different kinases all belonging to the conserved phosphatidylinositol 3-kinase related protein kinase (PIKK) superfamily (reviewed in $[5,6])$. These kinases transmit the signals from the site of DNA damage to the cell cycle machinery by activating cell cycle checkpoints. The G2/M DNA damage checkpoint is critical for the maintenance of genome stability as unrepaired DNA double strand breaks (DSB) may directly cause mistakes in chromosomal segregation 
to the daughter cells if ending up in the $M$ phase of the cell cycle. Hence, the final exit strategy in multicellular organisms before cellular transformation, and eventually cancer occurs as consequence of DDR is cellular suicide, i.e. apoptosis (reviewed in $[7,8]$ ).

The Ataxia-Telangiectasia Mutated (ATM) and ATM and Rad3-related (ATR) kinases are the central mediators of DDR (reviewed in $[5,6]$ ). ATM is known to orchestrate a global response to DSB in higher organisms including DNA repair, checkpoint activation and apoptosis. Well characterized targets of ATM are the Chk2 kinase and the tumour suppressor p53, the latter being stabilized upon DNA damage to further initiate specific target gene expression executing cell cycle arrest, DNA repair and apoptosis, respectively (reviewed in [1,9]). Whereas ATM is involved primarily in the mammalian DSB response, ATR is activated by a much wider range of genotoxic stresses and appears to be a much more important player in DDR of yeast cells than ATM (reviewed in $[2,6])$. Once activated, ATR phosphorylates and activates the protein kinase Chk1, which effects a cell cycle arrest at the G2/M transition, allowing more time for DNA repair so that cells do not enter mitosis prematurely. Noteworthy, there is considerable crosstalk between the ATM/ATR signal transduction pathways (reviewed in $[6,8]$ ).

The Drosophila homologue of ATM is called telomere fusion (tefu), as it was originally identified by its essential role in telomere maintenance [10]. Although tefu is important for p53 activation and DNA damage-induced apoptosis, it has no evident role in cell cycle arrest in response to DNA damage [10-14]. Similar to vertebrates, the Drosophila Chk2 homologue loki (lok) regulates p53-mediated apoptosis in response to DNA damage as well as to telomere loss [15-21]. The ATR homologue in Drosophila is encoded by meiotic 41 (mei-41). Mutational analyses revealed that mei-41 is indispensable for meiotic recombination checkpoints as well as for DNA damage checkpoints in somatic cells [13, 22-27]. Like its target kinase grapes ( $g r p$ ) (the Drosophila Chk1 homologue), mei-41 is important to postpone the mitosis entry in larval cells after IR-stress [24, 25, 28]. Moreover, mei-41 and grp mutant flies are highly sensitive towards triggers that damage DNA or inhibit DNA replication, and are therefore essential to maintain genomic and chromosomal stability [29-33]. Overall in Drosophila, mei-41 appears to mostly fulfil the roles of both ATM and ATR with regard to DDR, whereas tefu's primary role is the maintenance of telomeres and triggering apoptosis.

Although various mei-41 mutant alleles have been analyzed in the past in Drosophila, no gain-of-function allele is yet available. In order to fill this gap, we generated a mei-41 construct under UAS-control, which allows temporal and tissue-specific expression of mei-41 with the help of the versatile Gal4/UAS system [34]. We show that the overexpression of mei-41 in imaginal tissues is sufficient to induce a G2 arrest constraining the growth of affected tissues. Moreover, in the presence of ectopic Mei- 41 cells are hampered to resume the cell cycle after irradiation (IR)-mediated arrest. Upon IR-stress, Grp protein shows retarded mobility, and likewise upon mei-41 overexpression, suggesting that ectopic Mei-41 protein is sufficient to phosphorylate Grp protein. Finally, using a p53-biosensor we show that overexpression of mei-41 effects p53 reporter gene expression in vivo, suggesting a link to the Chk2/lok pathway as well. Overall, our data provide evidence for a mei-41-induced cellular response independent of DDR-mediated mei-41 activation.

\section{Results}

Generation of a mei-41 overexpression construct

Aiming for a deeper understanding of the Drosophila DNA damage response, we concentrated on meiotic 41 (mei-41), the Drosophila checkpoint kinase ATR homologue and key player of this process $[24,25]$. To study the consequences of mei-41 overexpression, we generated a pUAST-mei-41 construct allowing for tissue-specific induction during fly development with the help of the Gal4/UAS-system [34]. The mei-41 locus contains four small introns (59 bp, $57 \mathrm{bp}, 74 \mathrm{bp}, 64 \mathrm{bp}$ ) and covers more than $8 \mathrm{~kb}$; there is no complete cDNA available (7899 bp) (see http://flybase.org for further details). We therefore decided to PCR-amplify genomic DNA in four smaller fragments to be fused to cover the entire open reading frame, and clone it into the pUAST vector (Fig. 1a). Transgenic lines were established, tested and a third chromosomal insertion (3.3) line was used for further experiments. Overexpression of mei-41 in the posterior compartment of wing imaginal discs using en-Gal4-GFP was demonstrated by in situ hybridization with a mei-41 specific probe (Fig. 1b). Moreover, qRT-PCR revealed about a 500-fold induction when UAS-mei41 was ubiquitously induced with $d a-G a l 4$ during larval development relative to the endogenous mei-41 expression levels (Fig. 1c). We conclude that the UAS-mei-41 construct is well suited for overexpression studies during Drosophila development.

\section{Overexpression of mei-41 affects entry into mitosis}

Primary to DDR is checkpoint activation, i.e. slowing down the entry into mitosis to allow time for repair [5]. We wondered whether the overexpression of mei-41 without further activation by DNA damaging compounds might suffice to affect cell cycle regulation. To this end, we induced mei-41 in the posterior compartment of the wing disc using en-Gal4-GFP, and monitored cells in $M$ phase of the cell cycle (Fig. 2). Cells in mitotic phase were visualized with 


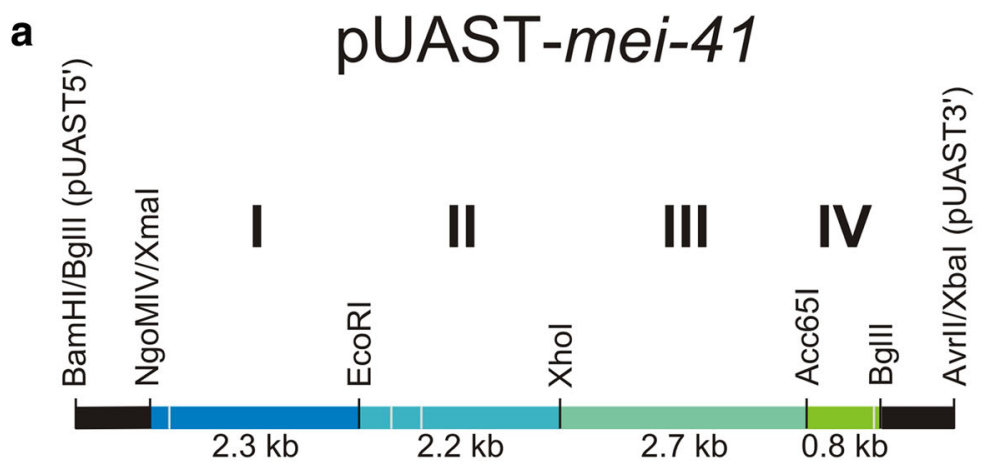

\section{b mei-41 probe}
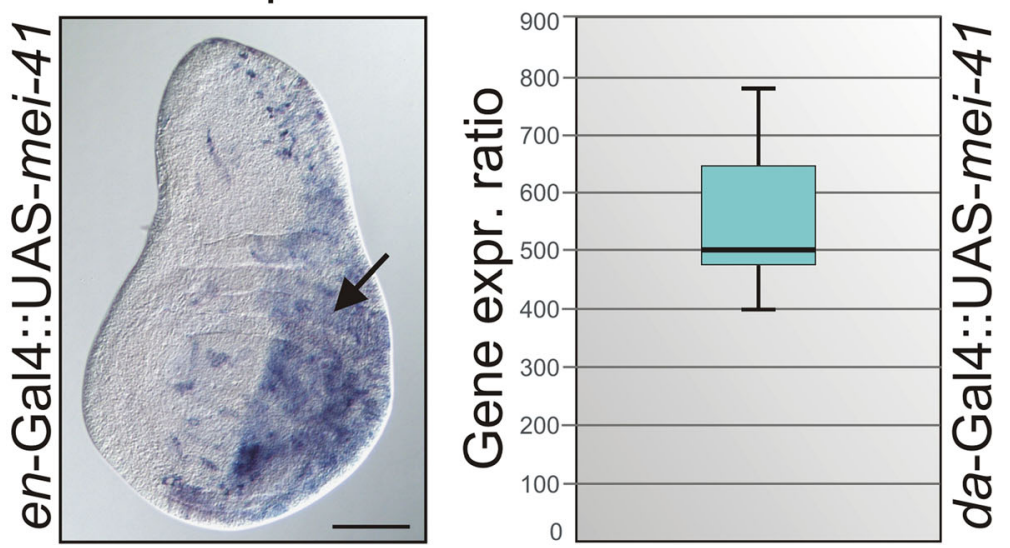

Fig. 1 Generation of a UAS-mei-41 overexpression line. (a) Cloning scheme of the pUAST-mei-41 construct. Coloured line corresponds to the mei-41 gene; intron positions are depicted as pale dashes. Segments derived from plasmid vectors are depicted in black and are not to scale. Genomic mei-41 DNA was PCR-amplified in fragments I-IV, which were eventually merged and shuttled into pUAST transformation vector in the correct 5'-3' orientation. (b) UAS-mei-41 flies were crossed to en-Gal4-GFP, and induction of mei-41 expression was visualized in the posterior compartment of a wing imaginal disc by in situ hybridization (arrow). Size bar represents $100 \mu \mathrm{m}$. (c) Overexpression of mei-41 was quantified by qRT-PCR. To this end, UAS-mei-41 was ubiquitously induced with da-Gal4, and mRNA isolated from third instar larvae. Compared to control (da::lacZ), mei-41 is about 500-fold overexpressed. Data were assembled from three biological and two technical replicates. Mini-max depicts $95 \%$ confidence, median corresponds to expression ratio. Gapdh and beta-Tubulin56D served as reference genes. Efficiencies for mei-41 (0.92), for gapdh (0.94) and for beta-Tubulin56D (0.95) were accounted for determining relative quantities [44]

anti-Phospho-Histone H3 (PH3) antibody staining and counted in the posterior compartment. Then their numbers were related to the whole wing disc size. As negative control, we overexpressed UAS-lacZ. Moreover, we included both downstream kinase Chk $1 / 2$ homologues grapes (grp) and loki (lok), since overexpression studies so far had not included cell cycle analyses in imaginal tissue $[10,16,18]$. It has been shown earlier that grp has a major role in the DNA replication checkpoint, whereas the primary role of lok is p53-mediated apoptosis in response to IR [16, 18, 19, 25, 28, 35].

Quantification of the results allowed us to uncover a significant reduction in the number of mitotic cells upon the overexpression of either mei-41 or grp, but not of $l o k$ (Fig. 2a-e). This is in agreement with earlier reports that mei-41 and grp are involved in cell cycle arrest in
Drosophila cells and tissues, with a minor contribution of lok [16, 18, 25, 28]. We confirmed that the reduced number of mitotic cells after mei-41 or grp overexpression were not a consequence of apoptosis, as no increase of cleaved Caspase- 3 activity was detected in the posterior compartment of the discs (see Additional file 1: Figure S1). Our results therefore demonstrate that both, mei-41 and grp, act on cell cycle regulation in a dose dependent manner, in contrast to lok. Our data conform to the requirements of mei-41 and grp in G2/M checkpoint function [24, 25]. Moreover, they demonstrate that our newly generated inducible mei-41 construct is a valuable tool for further analysis of mei-41 roles in a gain-of-function background, complementing the information gathered so far in a mei-41 loss-of-function background. 

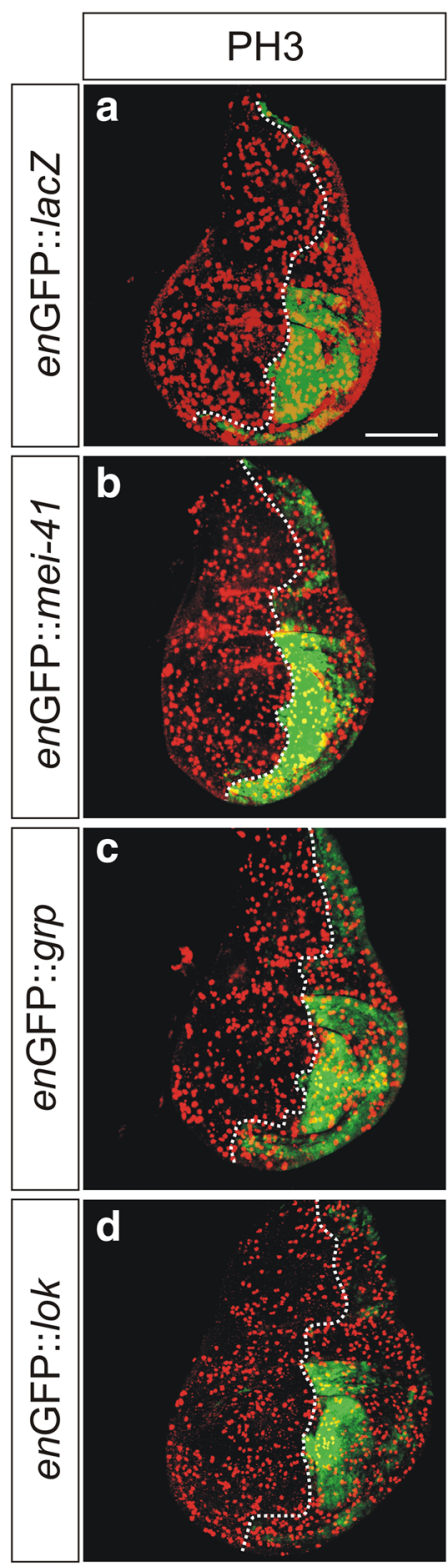

e

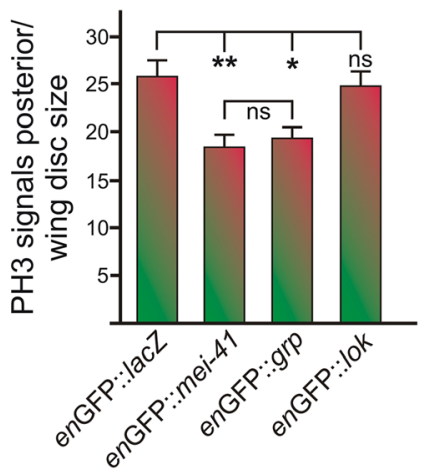

Fig. 2 Overexpression of mei-41 induces G2/M cell cycle arrest. (a-d) The checkpoint kinases mei-41, grp and lok (chk2) as indicated were overexpressed in the posterior compartment of wing imaginal discs using en-Gal4-GFP (green) (b-d). UAS-lacZ served as control (a). To image $\mathrm{M}$ phase, discs were stained with anti-Phospho-Histone $\mathrm{H} 3$ (PH3) antibodies (red) indicating the number of mitotic cells. GFP labelling was used to determine the antero-posterior boundary (dotted line). (e) Quantification of PH3 signals showed significant downregulation upon overexpression of mei-41 and grp but not of lok. Significance was tested by ANOVA two-tailed Tukey-Kramer approach (** $p<0.01 ;{ }^{*} p<0.05$; ns: not significant). Bars represent standard error (SEM) from 13 to 17 analyzed discs per genotype. Size bar represents $100 \mu \mathrm{m}$ in all panels

\section{Ectopic induction of mei-41 during imaginal development reduces adult tissue size}

To study the effects of mei-41 overexpression on adult tissue size, we ectopically induced mei-41 during proliferative phases of eye and wing development. Overexpression of mei-41 in the anterior part of the developing eye disc using ey-Gal4 resulted in a profound reduction of adult eye size (Fig. 3a). This growth defect is not restricted to the eye since overexpression of mei-41 in the posterior compartment of the developing wing disc using en-Gal4-GFP reduced its size within the adult wing significantly, whereas the size of the anterior compartment was unaffected (Fig. 3b). We did not observe any disturbance of wing and venation morphology, implying that growth but not differentiation or patterning of the tissue was affected (Fig. 3b).

So far, our data suggest that overexpression of mei-41 is sufficient for the induction of a cell cycle arrest without affecting development. The effect is very mild though, and only apparent in a quantitative approach, which may be explained by the lack of kinase activation by genotoxic stress. We cannot rule out the formal explanation, however, that the excessive amounts of Mei-41 kinase may generally interfere with other aspects of cell growth and proliferation. For example, ATM/ ATR kinases must be tightly regulated in order to prevent aberrant activation of DDR. It is thought that the availability of specific protein cofactors, required for kinase recruitment to DNA damage sites, restricts kinase activity. ATR forms a heterodimer with its obligate partner ATRIP during the sensing of DNA damage in the course of DDR (reviewed in $[6,8]$ ), and so does Drosophila Mei-41 with the ATRIP homologue Mus 304 [25]. We propose that Mus 304 may be limiting the effects of mei-41 overexpression, as the gene is expressed only about threefold of mei-41 (http://flybase.org).

\section{Irradiation induced G2/M checkpoint is extended in mei-41 overexpressing cells}

We know from the literature that mei-41 is indispensable for the irradiation induced $\mathrm{G} 2 / \mathrm{M}$ checkpoint $[13,22,25]$. 

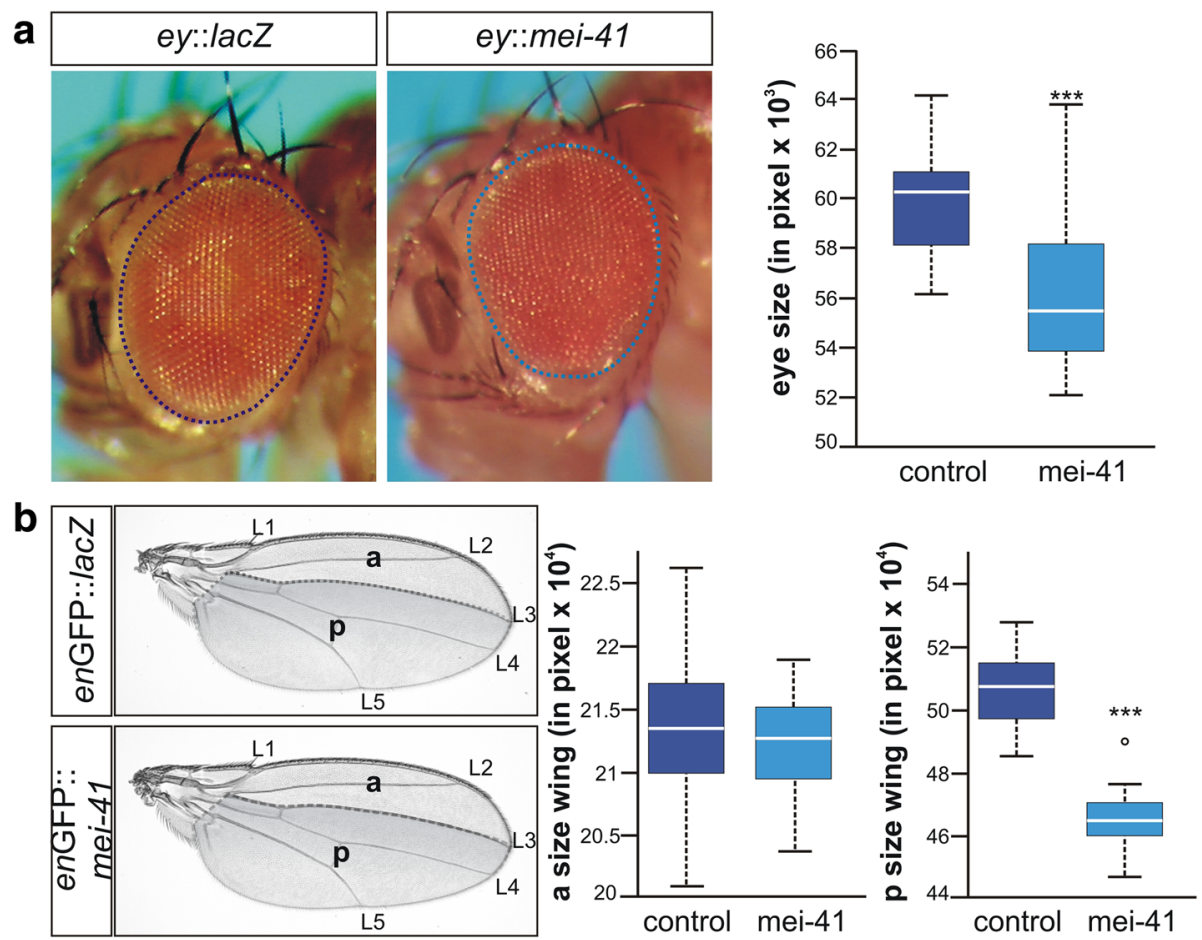

Fig. 3 Overexpression of mei-41 results in smaller adult tissue size. (a) UAS-mei-41 was overexpressed during eye development using ey-Gal4; likewise was UAS-lacZ serving as control. Eye size of adult female flies was measured as indicated. Compared to the control, mei-41 overexpression caused a significant decrease in eye size $(n \geq 26)$. (b) Effects on tissue size of the overexpression of UAS-mei-41 with en-Gal4-GFP in the posterior compartment of the developing wing were compared to those of the control UAS-lacZ $(n \geq 20)$. Size of anterior and posterior compartments was measured as indicated. Whereas no difference was detected in the anterior (a), the posterior compartment ( $p$, shaded grey) was significantly smaller when mei-41 was overexpressed. Longitudinal veins L1-L5 are labelled. Data are depicted as boxplots with center lines showing the medians, box limits indicate the 25th and 75th percentiles as determined by BoxPlotR software; whiskers extend 1.5 times the interquartile range. Statistics were done using ANOVA, and significance determined with Dunnett's approach $\left(^{* * *} p<0.001\right)$

Likewise, the combined activity of grp and lok is required, with a minor contribution of the latter [16, 18, 25, 28]. Accordingly, we wondered how overexpression of mei-41, grp or lok may affect the cellular response to irradiation. Larvae overexpressing the respective constructs in the posterior compartment of imaginal discs were exposed to 40 Gy IR. PH3 staining uncovered cells in early mitosis in wing imaginal discs: as expected, IR-induced a G2/M checkpoint as very few cells entered mitosis $1 \mathrm{~h}$ after irradiation (Fig. 4a, b). Cell cycle arrest is induced and maintained to allow sufficient time for DNA repair, and eventually the cell to recover from DNA damage and resume cell cycle. Accordingly, cells start to re-enter mitosis, seen by increasing numbers of $\mathrm{PH} 3$ positive cells over time. In the control, we observed rising numbers of PH3 positive cells already at $4 \mathrm{~h}$ post-IR, becoming considerably numerous $6 \mathrm{~h}$ later (Fig. 4a, b). A likewise increase was observed in the anterior compartment of the irradiated discs from larvae overexpressing either mei-41, grp or lok. In the posterior compartment, however, i.e. in cells overexpressing mei-41, re-entry into the cell cycle was considerably hampered even after $6 \mathrm{~h}$ recovery time (Fig. 4a, b), presumably by keeping the DSB damage checkpoint active. We expect both, the endogenous as well as the overexpressed Mei-41 kinase to be activated in response to IR-stress. Consequently, cells are expected to be flooded by activated Mei-41, keeping them from resuming mitosis entry. Even several hours post-IR, activated Mei-41 levels apparently surpass the threshold for a G2/M checkpoint.

Overexpression of grp had a much milder effect: $4 \mathrm{~h}$ after irradiation, cell cycle had not yet resumed, whereas after $6 \mathrm{~h}$, the number of mitotic cells was approaching that of the control (Fig. 4a, b). These data indicate that grp overexpression slowed down cell cycle re-entry, but not as efficiently as mei-41 overexpression. This result may be expected, since grp encodes the effector kinase downstream of mei-41: IR-stress activates endogenous Mei-41 kinase which phosphorylates and activates the effector kinase Grp. In the presence of abundant Grp protein, this response will be intensified and longer lasting. Therefore, we expect cell recovery to lag behind, i.e. $g r p$ to mirror but not to match mei-41 overexpression. 

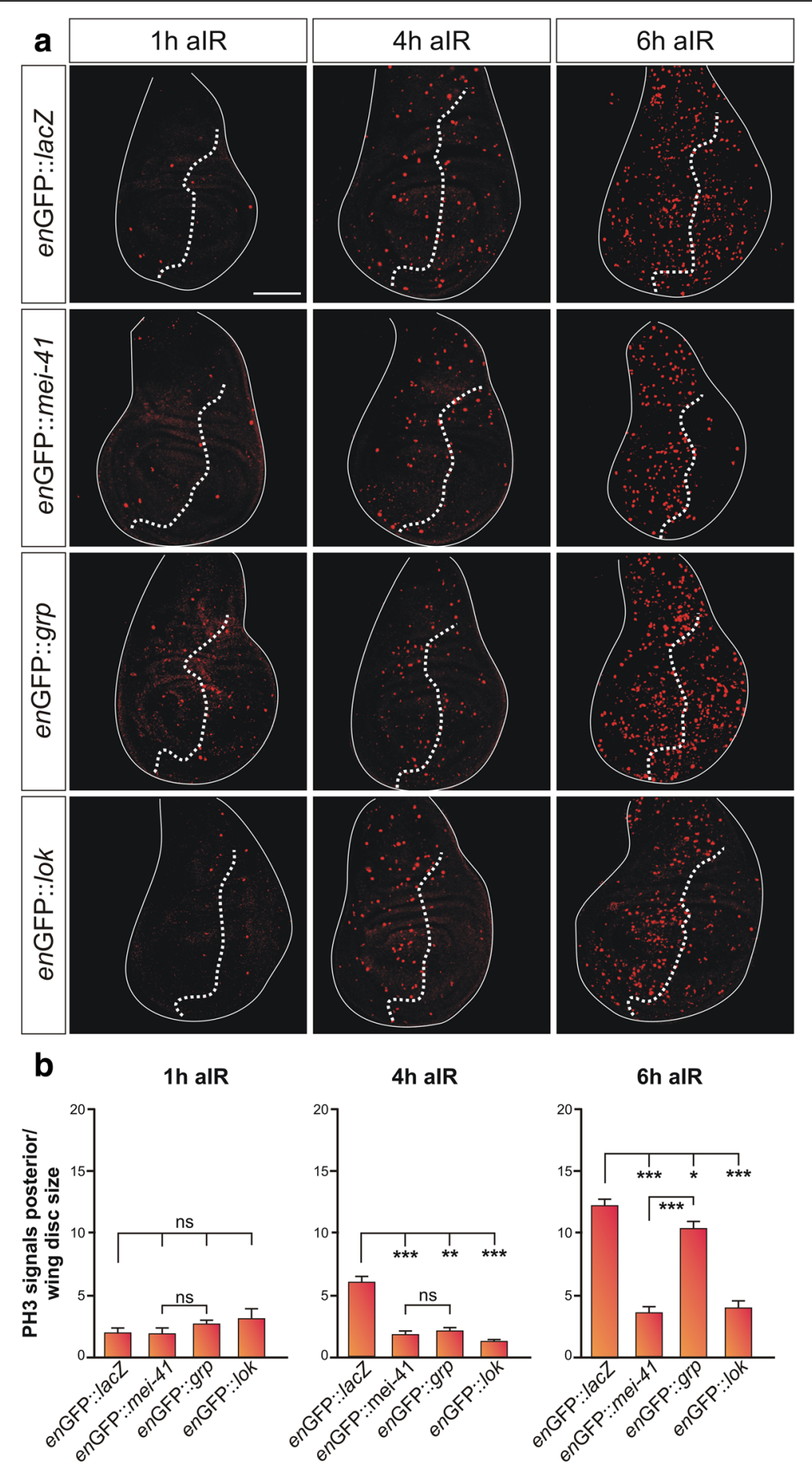

Fig. 4 (See legend on next page.) 
(See figure on previous page.)

Fig. 4 Cell cycle re-entry is delayed in cells overexpressing mei-41. (a) The indicated kinase or the lacZ control was overexpressed in the posterior compartment of wing imaginal discs using en-Gal4-GFP. Third instar larvae of the given genotypes were irradiated with 40 Gy and allowed to recover for one, four and six hours ( 1 h, 4 h, 6 h alR) respectively, before dissection of the wing imaginal discs. Mitotic cells are highlighted by Phospho-Histone H3 antibody staining (red). GFP labelling was used to determine the antero-posterior boundary (indicated by the dotted line). IR-stress caused a cell cycle arrest, only few cells appear in M phase one hour after irradiation. Entry into mitosis reappears with time in the control (enGFP:./acZ), as well as in the anterior compartment of all genotypes as DNA damage response proceeds. Not so in the posterior compartment, though, where either mei-4l or lok is overexpressed. However, overexpression of grp allowed the cell cycle to resume $6 \mathrm{~h}$ post-IR. Size bar represents $100 \mu \mathrm{m}$ in all panels. (b) Quantification of PH3 signals in the posterior compartment reveals no significant difference amongst the different genotypes $1 \mathrm{~h}$ after irradiation (alR). Re-entry into mitosis is observed in the wild type but not the other genotypes at $4 \mathrm{~h}$ alR. Even at $6 \mathrm{~h}$ alR, significantly less mitotic cells are observed in the posterior compartments overexpressing either mei-41 or lok, whereas grp overexpression had only minor lasting effects. ${ }^{* * *} p<0.001 ;{ }^{* *} p<0.01 ;{ }^{*} p<0.5$; ns, not significant) according to ANOVA two-tailed Tukey-Kramer approach for multiple comparisons. Bars represent standard of the mean (SEM). At least 16 wing discs were analyzed for each genotype and time point

Overexpression of lok, however, had a similar effect as mei-41 overexpression (Fig. 4a, b), which, at first glance, came as a surprise: cell cycle re-entry appeared delayed even at $6 \mathrm{~h}$ after irradiation, i.e. the number of PH3 positive cells was still reduced. However, we had not observed any effect of lok overexpression on cell cycle regulation in the absence of IR-stress (Fig. 2a), in agreement with the many reports on the supplemental role of lok for the G2/M checkpoint [25, 28]. Instead, the specific role for lok in the activation of p53-mediated cell death is well established $[15,16,25]$. Thus, we wondered whether the lack of $\mathrm{PH} 3$ positive lok overexpressing cells several hours post-IR indeed reflected failure of cell cycle re-entry, or alternatively might be explained by IR-induced lok-mediated apoptosis. To this end, although no signs of cell death were observed in unstressed discs where lok was overexpressed (Fig. 2), we repeated the experiment, now addressing apoptosis induction by IR-stress with $6 \mathrm{~h}$ recovery time. Indeed, in response to IR-stress accumulation of cleaved Caspase- 3 was detected most prominently in the central part of the wing disc in all genotypes (see Additional file 1: Figure S2). In contrast to the discs overexpressing either lacZ, mei-41 or grp, however, lok overexpressing cells showed a much stronger apoptotic response than any of the others (see Additional file 1: Figure S2). We conclude that the lower numbers of $\mathrm{PH} 3$ positive cells present in lok overexpressing tissue may result primarily from an increase in apoptosis rather than from a delay of cell cycle re-entry.

\section{Mobility shift of Grp after mei-41 overexpression}

Using Drosophila Schneider S2 cells, it has been demonstrated that Grp is phosphorylated in response to DNA damage or incomplete DNA replication, and that this phosphorylation was dependent on the presence of Mei-41 [28]. Presumably, the Drosophila ATR homologue Mei-41 phosphorylates Grp in the process of DDR, comparable to what is known from other organisms like yeast, Хenopus and also mammals [2,3]. As we had seen a dose dependency for mei-41 on checkpoint induction (Fig. 2), we wondered, whether the sole overexpression of mei-41 may suffice to induce Grp phosphorylation, i.e. induce a bona fide DNA damage response. To this end, mei-41 was ubiquitously overexpressed together with a HA-tagged form of Grp using da-Gal4. Larval protein extracts from imaginal discs were separated by Phos-Tag ${ }^{\text {Tax }}$ PAGE to increase the separation of phosphorylated from unphosphorylated proteins, followed by Western blotting. For negative control, larvae just overexpressing HA-tagged Grp were used; for positive control such larvae had been subjected to 40 Gy IR-stress. Indeed, overexpression of mei-41 resulted HA-tagged Grp to migrate more slowly compared to the negative control, but similar to the one detected $1 \mathrm{~h}$ post-IR (Fig. 5). These results suggest, that overexpression of mei-41 is sufficient to induce DDR at low levels, i.e. to activate, respectively phosphorylate its downstream target Grp in vivo.

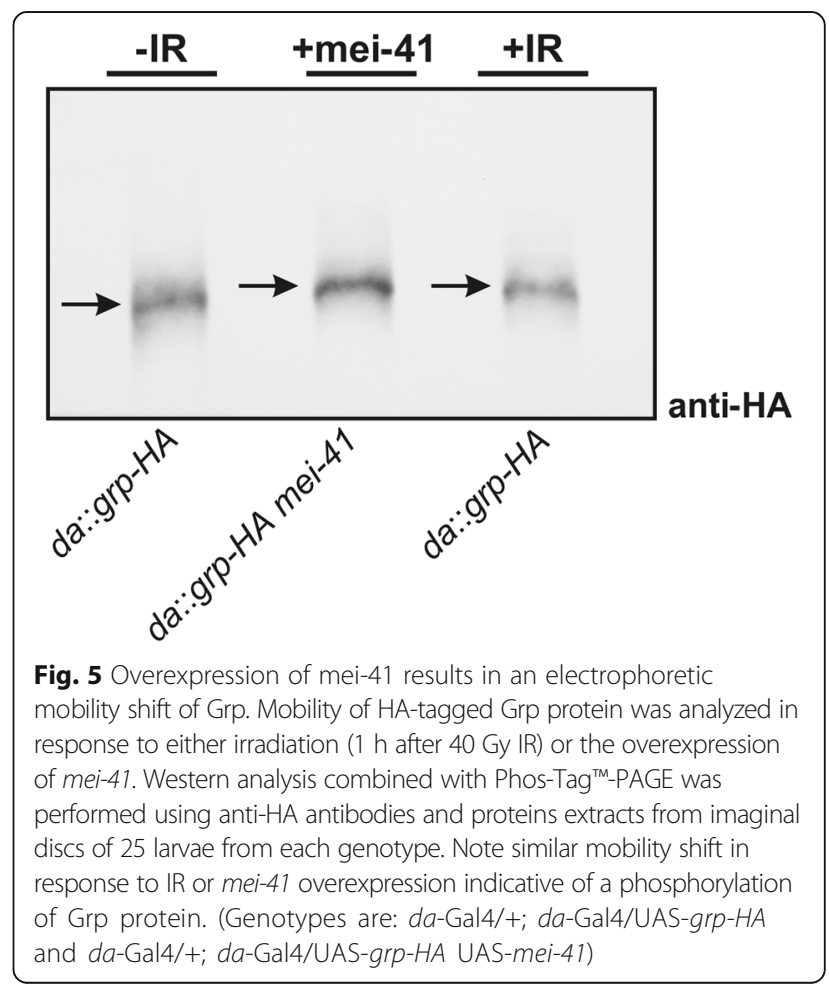




\section{Activation of the p53R-GFP biosensor can be achieved by overexpression of mei-41}

As outlined above, mei-41 mutants display checkpoint defects that are only matched by grp; lok double mutants, implying that Mei-41 may not only act on Grp but also, to a lesser degree, on $\operatorname{Lok}[16,25,28]$. Genetic and molecular data imply that Lok, but not Grp, is an activator of p53 in response to DNA damage in Drosophila $[16,25]$. To investigate a potential cross-regulatory effect of the Drosophila ATR homologue Mei-41 on p53 activity we made use of a p53R-GFP biosensor, where p53 activation is reflected by nuclear GFP accumulation [36]. This p53R-GFP biosensor is activated during meiosis in the female germline, as well as in response to genotoxic stress in somatic tissues [36-38]. Thus, it is well suited to determine whether mei-41 overexpression alone may suffice to effect p53 activation. UAS-mei-41 was ubiquitously overexpressed with da-Gal4 in the background of the p53R-GFP reporter, and the giant salivary gland nuclei were examined for accumulation of GFP. GFP signal intensity was measured, using nuclear Putzig (Pzg) protein [39] as internal standard. For control, p53R-GFP nuclear localization was evaluated after ectopic induction of either Lok or Grp as well. As expected from its ability to activate p53 [16, 18, 19], overexpression of lok caused a strong nuclear GFP signal, which was well above lacZ control, demonstrating the reliability of our test system (Fig. 6a, b). Interestingly, overexpression of mei-41 was sufficient to induce nuclear p53R-GFP accumulation, albeit much weaker than that of lok (Fig. 6a, b). In contrast, Grp was unable to trigger measurable GFP signals (Fig. 6a, b). In addition to the visual assessment, qRT-PCR measurements of GFP expression levels were conducted on larval imaginal discs. They uncovered a 27-fold and 5-fold increase of p53-GFP reporter expression, respectively, in response to ubiquitous lok and mei-41 overexpression in comparison to the lac $Z$ control (Fig. 6c). These data provide unambiguous evidence that ectopic Mei-41 is able to induce p53 activity. Whether this activation is direct, as shown for ATM as well as ATR (reviewed in $[6,8]$ ), or indirect via Lok, requires further investigations. Our newly established UAS-mei-41 construct is well suited to facilitate future analyses.
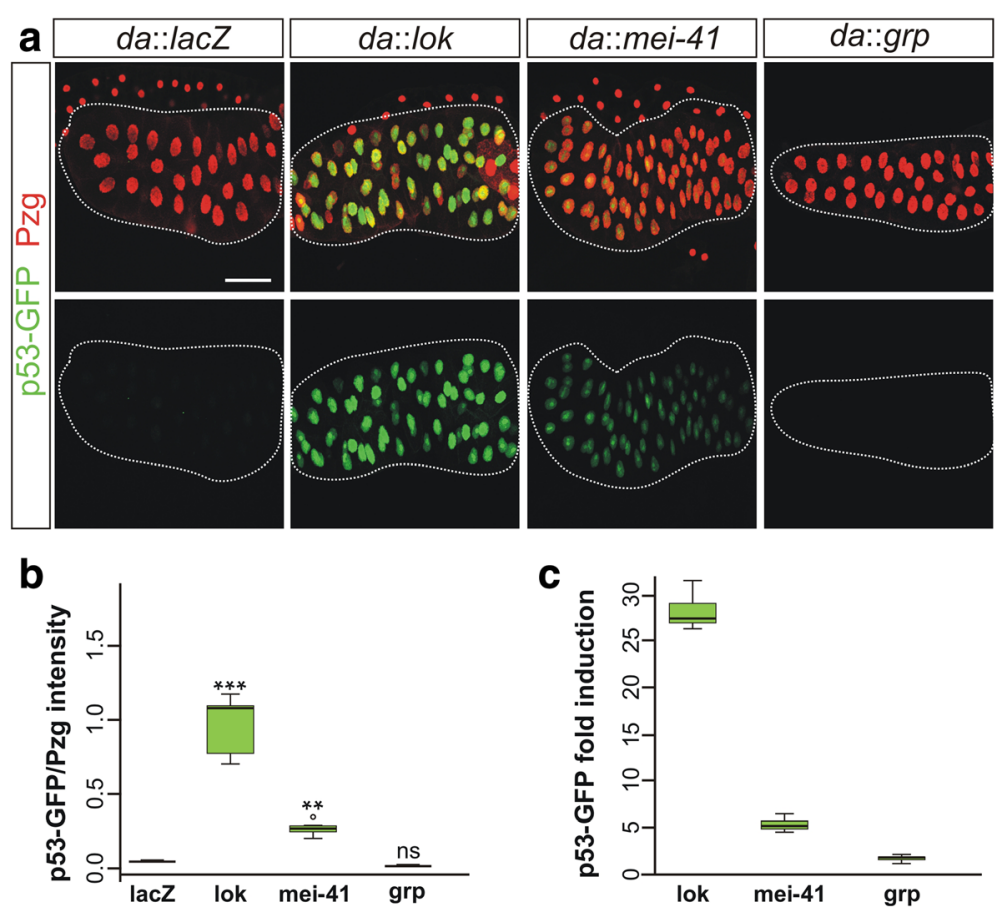

Fig. 6 Response of the p53R-GFP reporter on mei-41 overexpression. (a) In response to p53 activation, nuclear GFP is expressed from the p53RGFP reporter [36]. This system was used to assay p53 activation in consequence of the overexpression of either lok, mei-41 or grp; lacZ served as control. Salivary glands were analyzed; their nuclei visualized with the nuclear marker Pzg [39]. In contrast to lacZ and grp, overexpression of lok and to a lesser degree mei-41 resulted in a robust induction of the p53R-GFP reporter. Size bar represents $100 \mu \mathrm{m}$ in all panels. (b) Quantification of nuclear p53R-GFP intensity was determined relative to the mean intensity of the nuclear marker protein Pzg ( $n=84)$. Induction of lok strongly induced p53R-GFP nuclear accumulation. Also mei-41 caused a significant nuclear accumulation of p53R-GFP, whereas grp did not. *** $p<0.001$; ** $p<0.01$; ns. not significant according to ANOVA two tailed Dunnet's approach. (c) Expression levels of GFP were quantified by qRT-PCR in third instar larvae. Overexpression of lok and mei-41 with da-Gal4 considerably increased p53R-GFP reporter gene activity in relation to the lacZ-control: lok 27.5-fold, mei-41 4.9-fold and grp 1.6-fold. Data were assembled from four biological and two technical replicates. Mini-max depicts 95\% confidence, median corresponds to expression ratio. As reference genes, cyp33 and Tbp were used. Efficiencies for GFP (0.91), for cyp33 (0.96) and Tbp (0.95) were accounted for in determining relative quantities [44] 


\section{Discussion}

In this work, we show that mei-41 and grp act on cell cycle regulation in a dose dependent manner, conforming to the requirements of mei-41 and grp in G2/M checkpoint function [24, 25]. Whereas developmental patterning is unaffected, a cell cycle arrest and proliferation defects result from the overexpression of mei-41. Moreover, we unambiguously show that p53 activity is induced by ectopic Mei-41. We do not know, however, whether this activation is direct, as shown for ATM as well as ATR (reviewed in $[6,8]$ ), or indirect via Lok. The defects resulting from Mei-41 overexpression are very mild, however, both regarding cell cycle delay, cell proliferation or apoptosis, compared to a normal irradiation response. There are several explanations. For example, during genotoxic stress Mei-41 kinase is activated by phosphorylation, which does not follow the overexpression. Moreover, Mei-41 heterodimerizes with its obligate partner Mus 304 [25] during damage response [6, 8]. In our overexpression experiments, Mus 304 may be limiting, allowing only for the mild effects observed. Finally, excessive amounts of Mei-41 kinase resulting from the overexpression may generally interfere with other aspects of cell growth and proliferation.

\section{Conclusions}

Overexpression of the Drosophila ATR homologue $m e i-41$ during imaginal development is sufficient to initiate a cellular response resembling the DNA damage response, which is reflected by the induction of a G2/M checkpoint, growth retardation, apparent phosphorylation of the effector kinase Grp (Drosophila Chk1), as well as the activation of $\mathrm{p} 53$. The rather subtle effects presumably result from a lack of Mei-41 kinase activation by genotoxic stress, as IR resulted in long-lasting checkpoint activation. Moreover, the observation of $\mathrm{p} 53$ activation in response to mei-41 overexpression indicates cross-talk of ATR/ATM pathways also in Drosophila that may involve the effector kinase Lok (Drosophila Chk2).

\section{Methods \\ Cloning of pUAST-mei-41 and generation of transgenic flies}

Four fragments covering mei-41 were PCR-amplified from genomic DNA, and subcloned individually in $\mathrm{pBT}$ vector (Stratagene, La Jolla, USA) (fragments I-III) or pGX-attP [40] (fragment IV) to obtain the following subclones: pBT $2.3 \mathrm{~kb}$ NgoMIV/XmaI - EcoRI fragment I (translation start to EcoRI); pBT $2.2 \mathrm{~kb}$ EcoRI-XhoI fragment II; pBT $2.7 \mathrm{~kb}$ XhoI-Acc65I fragment III; pGX-attP $0.8 \mathrm{~kb}$ Acc65I - BglII fragment IV (translation stop). Subsequently fragments I-III were fused by successive cloning in pBT vector. The insert was excised as SacII/Acc65I fragment and cloned into likewise opened pGX-attP harbouring fragment IV. The full length genomic mei-41 construct was excised with BamHI/AvrII and subsequently shuttled into $B g l \mathrm{II} / \mathrm{XbaI}$ of pUAST vector [34] and sequence verified. Three independent transgenic fly lines were established by P-element mediated germline transformation [41]. All three lines were functionally tested for ectopic expression of mei-41 RNA by in situ hybridization of imaginal discs. The subsequent functional assays were performed with line UAS-mei-41 (3.3) inserted on the third chromosome.

RNA expression analyses: In situ hybridization and qRT-PCR In situ hybridization on larval wing discs was performed with digoxigenin-labelled DNA probes of mei-41 according to standard protocols [42]. Quantitative RT-PCR was performed as outlined earlier [38, 43]. With the PolyATract $^{\oplus}$ System 1000 kit (Promega Mannheim, Germany) $\operatorname{poly}(\mathrm{A})^{+}$RNA was isolated from 20 total third instar larvae for the quantification of UAS-mei-41 expression, and from imaginal discs only attached to the mouth hook from 20 third instar larvae for p53R-GFP biosensor quantification. Real time qPCR was conducted with Blue S'Green qPCR kit (Biozym, Hessisch-Oldendorf, Germany) on $6 \mathrm{ng}$ of cDNA in $10 \mu \mathrm{l}$ end volume using MIC magnetic induction cycler (bms, Pots Point, Australia) including target and no-template controls. Absence of genomic DNA was tested in a non-RT control. As internal references for mei-41 expression, $\beta T$ Tub56D and gapdh2 were used, whereas cyp33 and Tbp served as internal references in the case of $p 53 R$-GFP biosensor quantification. The references were selected based on variance and $\mathrm{Cq}$ values. Relative quantification of the data was performed with micPCR software Version 2.6.0 based on REST taking target efficiency into account [44]. At least three biological and two technical replicates were performed. The following primer pairs were used $\left(5^{\prime}->3^{\prime}\right)$ :

$\begin{array}{ll}\text { mei-41 } & \text { upper, CTC CTG CAA GAC TTT AAT } \\ & \text { TCG CTC AC } \\ & \text { lower, GCG TTG GCT GCA TGT ACT } \\ & \text { TCT CA } \\ \text { BTub56D } & \text { PP17563 DRSC FlyPrimer bank [45] } \\ \text { gapdh2 } & \text { PP2976 DRSC FlyPrimer bank [45] } \\ \text { GFP } & \text { upper, TCAAGGACGACGGCAACTA } \\ & \text { CAAGAC } \\ & \text { lower, TCACCTTGATGCCGTTCTT } \\ & \text { CTGC } \\ \text { cyp33 } & \text { PP14577 DRSC FlyPrimer bank [45] } \\ \text { Tbp } & \text { PP1556 DRSC FlyPrimer bank [45] }\end{array}$

Fly work and immunochemistry

Flies were raised on standard corn-molasses food at $25^{\circ} \mathrm{C}$. The following strains were used: da-Gal4 (BL55849); en-Gal4-GFP [46], UAS-lacZ (BL8530), UAS-mei-41 (this 
work), UAS-grapes.ORF.3xHA (F000934; obtained from FlyORF; Zürich, Switzerland) [47], UAS-chk2 (lok) (gift of U. Abdu) [48], UASp-lacZ [49], p53R-GFP [36]. Staining of third instar wing imaginal discs or salivary glands was done according to standard protocols as described earlier $[38,50]$ using the following antibodies: rabbit anti-GFP (1:100; Santa Cruz Biotech, Dallas, USA), guinea pig anti-Pzg (1:1000) [39], rabbit anti-cleaved Caspase-3 (1:250; Cell Signaling, Germany) and rabbit anti-Phospho-Histone H3 (PH3) (1:50; Cell Signaling, Germany). Secondary antibodies from goat or donkey, coupled to FITC or Cy3, were obtained from Jackson Immuno-Research Laboratories (Dianova, Hamburg, Germany). Larval tissue was documented by confocal microscopy using a MRC1024 confocal scan head coupled to a Zeiss Axiophot (Carl Zeiss AG, Oberkochen, Germany) and LaserSharp 2000 imaging software. Pictures were compiled with Corel Photo Paint and Corel Draw software.

\section{lonizing radiation (IR) treatment and Phos-Tag ${ }^{\mathrm{TM}}$ based mobility shift detection}

Third instar larvae were irradiated with 40 Gy using Elektra Versa HD linear accelerator (Elektra Instrument AB; Stockholm; Sweden) at the Marienhospital Stuttgart. To investigate phosphorylation of Grp protein, 25 third instar larvae of the genotype da-Gal4/+; da-Gal4/ UAS-grp-HA were irradiated with 40 Gy. After $1 \mathrm{~h}$ recovery time, imaginal discs connected to the mouth hook were isolated and homogenized in $50 \mu \mathrm{l}$ binding buffer (20 mM HEPES pH 7.6, $150 \mathrm{mM} \mathrm{KCl,} 2.5 \mathrm{mM}$ $\mathrm{MgCl}_{2}, 10 \%$ glycerol, 0.05\% NP-40, 1 mM DTT, ROCHE complete ULTRA protease inhibitor mini tablet). The unirradiated controls as well as the $d a-G a l 4 /+; d a-G a l 4 /$ UAS-grp-HA UAS-mei-41 larval tissues were treated likewise. The homogenates were separated in $10 \%$ SDS-PAGE including $50 \mu \mathrm{M}$ Phos-Tag ${ }^{\text {TM }}$ Acrylamide solution (\#AAL-7, Wako Chemicals GmbH, Neuss, Germany) and $100 \mu \mathrm{M} \mathrm{MnCl}$ at $70 \mathrm{~V}$ for $22-24 \mathrm{~h}$ at $8{ }^{\circ} \mathrm{C}$. After blotting on PVDF membrane (BioRad, Munic, Germany), HA-tagged Grapes was detected with rat anti-HA (1:2500, Roche Diagnostic, Basel, Switzerland), and secondary anti-rat antibody coupled to alkaline phosphatase (1:1000; Jackson Immuno-Research Laboratories via Dianova, Hamburg, Germany).

\section{Documentation and statistical evaluation of larval and adult tissue}

Cells in $\mathrm{M}$ phase within the posterior compartment were counted based on PH3 signals. The posterior compartment was determined by GFP labelling from en-Gal4-GFP [46]. Cell number was related to total size of the respective wing discs using ImageJ. p53R-GFP expression in salivary glands was examined by measuring signal intensity of 12 nuclei from seven different glands each ( $n=84$ nuclei), using the mean intensity of Pzg signals as internal standard. Wings from female flies were dehydrated in ethanol, mounted in Euparal (Roth, Karlsruhe, Germany) and documented with an ES120 camera (Optronics, Goleta CA, USA) connected to a Zeiss Axiophot (Carl Zeiss AG, Jena, Germany) using Pixera Viewfinder software, version 2.0. Female flies were etherized before taking pictures from the heads with an ES120 camera coupled to a Leica Wild M3C Stereomicroscope (Leica, Wetzlar, Germany). Size of female eyes (UASp-lacZ/+; ey-Gal4/+ and ey-Gal4/+; UAS-mei-41/+) or wings (UASp-lacZ/+; en-Gal4-GFP/+ and en-Gal4-GFP/+; UAS-mei-41/+) was measured using Image $J$. Statistical analysis was conducted by ANOVA using a two-tailed Tukey-Kramer or Dunnett's test for multiple comparisons. ${ }^{* * * *} p<0.001$ highly significant; ${ }^{* *} p<0.01$ very significant; $p<0.05$ significant; not significant (ns) $p>0.05$. Box plots were compiled using the online plotting tool BoxPlotR (http://shiny.chemgrid.org/boxplotr/).

\section{Additional file}

Additional file 1: Analysis of apoptosis. (PDF 9148 kb)

\begin{abstract}
Abbreviations
ATM: Ataxia-Teleangiectasia Mutated; ATR: ATM and Rad3-related; Chk1, Chk2: Checkpoint kinase 1, 2; da: Daughterless; DDR: DNA damage response; DSB: DNA double strand breaks; en: Engrailed; GFP: Green fluorescent protein; grp: Grapes; IR: Ionizing radiation; lok: Loki; mei-41: Meiotic 41; PH3: PhosphoHistone $\mathrm{H3}$
\end{abstract}

\section{Acknowledgements}

We thank C. Gromoll from the Marienhospital Stuttgart for allowing us to use the Elektra Versa HD linear accelerator for our IR experiments. We are indebted to U. Abdu, the Bloomington Stock Center (Bloomington, USA), the Developmental Studies Hybridoma Bank (DSHB) and the FlyORF collection

(Zürich, Switzerland) for fly lines and antisera.

\section{Funding}

This work was supported by grants from the German Science Foundation to ACN (NA 427/4-1) and AP (PR 533/10-1). The funders had no role in study design, data collection and analysis, decision to publish or preparation of the manuscript.

\section{Availability of data and materials}

The datasets supporting the conclusions of this article are included within the article and its additional information file. Materials are available from the corresponding author on reasonable request.

\section{Authors' contributions}

ACN and AP conceived and designed the experiments; FEB, MZ and ACN conducted the experiments, FEB, MZ, AP and ACN collected and analyzed the data and performed the statistical analysis; ACN and AP wrote the manuscript; and all authors have approved the final manuscript.

Ethics approval and consent to participate Not applicable.

Consent for publication

Not applicable. 


\section{Competing interests}

The authors declare that there are no competing financial, personal, or professional interests.

\section{Publisher's Note}

Springer Nature remains neutral with regard to jurisdictional claims in published maps and institutional affiliations.

\section{Received: 23 July 2018 Accepted: 28 August 2018 Published online: 06 September 2018}

\section{References}

1. Zhou BB, Elledge SJ. The DNA damage response: putting checkpoints in perspective. Nature. 2000;408:433-9.

2. Melo J, Toczyski D. A unified view of the DNA-damage checkpoint. Curr Opin Cell Biol. 2002;14:237-45.

3. Song YH. Drosophila melanogaster: a model for the study of DNA damage checkpoint response. Mol Cells. 2005;19:167-79.

4. Shaltiel IA, Krenning L, Bruinsma W, Medema RH. The same, only different DNA damage checkpoints and their reversal throughout the cell cycle. J Cell Sci. 2015;128:607-20.

5. Abraham RT. Cell cycle checkpoint signaling through the ATM and ATR kinases. Genes Dev. 2001;15:2177-96.

6. Blackford AN, Jackson SP. ATM, ATRm and DNA-PK: the trinity at the heart of the DNA damage response. Mol Cell. 2017;66:801-17.

7. Nyberg KA, Michelson RH, Putnam CW, Weinert TA. Toward maintaining the genome: DNA damage and replication checkpoints. Annu Rev Genet. 2002; 36:617-56.

8. Lindsey-Boltz FA. Bringing it all together: coupling excision repair to the DNA damage checkpoint. Photochem Photobiol. 2017;93:238-44.

9. Shiloh Y. The ATM-mediated DNA-damage response: taking shape. Trends Biochem Sci. 2006;31:402-10.

10. Oikemus SR, McGinnis N, Queiroz-Machado J, Tukachinsky H, Takada S, Sunkel CE, Brodsky MH. Drosophila atm/telomere fusion is required for telomeric localization of HP1 and telomere position effect. Genes Dev. 2004; 18:1850-61.

11. Silva E, Tiong S, Pedersen M, Homola E, Royou A, Fasulo B, Siriaco G, Campbell SD. ATM is required for telomere maintenance and chromosome stability during Drosophila development. Curr Biol. 2004; 14:1341-7.

12. Song $Y H$, Mirey G, Betson M, Haber DA, Settleman J. The Drosophila ATM ortholog, AATM, mediates the response to ionizing radiation and to spontaneous DNA damage during development. Curr Biol. 2004;14: 1354-9.

13. Bi X, Gong M, Srikanta D, Rong YS, Drosophila ATM. Mre11 are essential for the G2/M checkpoint induced by low-dose irradiation. Genetics. 2005;171: 845-7.

14. Pedersen M, Tiong S, Campbell SD. Molecular genetic characterization of Drosophila ATM conserved functional domains. Genome. 2010;53: 778-86.

15. Xu J, Xin S, Drosophila DW. Chk2 is required for DNA damage-mediated response and apoptosis. FEBS Lett. 2001;508:394-8.

16. Peters M, DeLuca C, Hirao A, Stambolic V, Potter J, Zhou L, et al. Chk2 regulates irradiation-induced, p53-mediated apoptosis in Drosophila. Proc Natl Acad Sci U S A. 2002:99:11305-10.

17. Masrousha N, Yang L, Hijal S, Larochelle S, Suter B. Drosophila chk2 gene loki is essential for embryonic DNA double-strand break checkpoints induced in S phase or G2. Genetics. 2003;163:973-82.

18. Brodsky MH, Weinert BT, Tsang G, Rong YS, McGinnis NM, Golic KG, et al. Drosophila melanogaster MNK/Chk2 and p53 regulate multiple DNA repair and apoptotic pathways following DNA damage. Mol Cell Biol. 2004;24: 1219-31.

19. Wichmann A, Jaklevic B, Su TT. lonizing radiation induces caspase dependent but Chk2- and p53-independent cell death in Drosophila melanogaster. Proc Natl Acad Sci U S A. 2006;103:9952-7.

20. Titen SW, Golic KG. Telomere loss provokes multiple pathways to apoptosis and produces genomic instability in Drosophila melanogaster. Genetics. 2008;180:1821-32

21. Kurzhals RL, Titen SWA, Xie HB, Golic KG. Chk2 and p53 are haploinsufficient with dependent and independent functions to eliminate cells after telomere loss. PLoS Genet. 2011;7(6):e1002103.
22. Hari KL, Santerre A, Sekelsky JJ, McKim KS, Boyd JB, Hawley RS. The mei-41 gene of $D$. melanogaster is a structural and functional homolog of the human ataxia telangiectasia gene. Cell. 1995;82:815-21.

23. Ghabrial A, Schüpbach T. Activation of a meiotic checkpoint regulates translation of Gurken during Drosophila oogenesis. Nat Cell Biol. 1999;1:354-7.

24. Sibon OC, Laurencon A, Hawley R, Theurkauf WE. The Drosophila ATM homologue Mei-41 has an essential checkpoint function at the midblastula transition. Curr Biol. 1999;9:302-12.

25. Brodsky MH, Sekelsky JJ, Tsang G, Hawley RS. Rubin GM. mus304 encodes a novel DNA damage checkpoint protein required during Drosophila development. Genes Dev. 2000;14:666-78.

26. LaRocque JR, Jaklevic B, Su T, Sekelsky J. Drosophila ATR in double-strand break repair. Genetics. 2007;175:1023-33.

27. Joyce EF, Pedersen M, Tiong S, White-Brown SK, Paul A, Campbell SD, McKim KS. Drosophila ATM and ATR have distinct activities in the regulation of meiotic DNA damage and repair. J Cell Biol. 2011;195:359-67.

28. de Vries HI, Uyetake L, Lemstra W, Brunsting JF, Su TT, Kampinga HH, Sibon OC. Grp/DChk1 is required for G2-M checkpoint activation in Drosophila S2 cells, whereas Dmnk/DChk2 is dispensable. J Cell Sci. 2005;118:1833-42.

29. Banga SS, Shenkar R, Boyd JB. Hypersensitivity of Drosophila mei-41 mutants to hydroxyurea is associated with reduced mitotic chromosome stability. Mutat Res. 1986;163:157-65.

30. Yu KR, Saint RB, Sullivan W. The grapes checkpoint coordinates nuclear envelope breakdown and chromosome condensation. Nat Cell Biol. 2000;2:609-15.

31. Jaklevic BR, Su TT. Relative contribution of DNA repair, cell cycle checkpoints, and cell death to survival after DNA damage in Drosophila larvae. Curr Biol. 2004:14:23-32

32. Jaklevic B, Uyetake L, Lemstra W, Chang J, Leary W, Edwards A, et al. Contribution of growth and cell cycle checkpoints to radiation survival in Drosophila. Genetics. 2006;174:1963-72.

33. Dekanty A, Barrio L, Milan M. Contributions of DNA repair, cell cycle checkpoints and cell death to suppressing the DNA damage-induced tumorigenic behavior of Drosophila epithelial cells. Oncogene. 2015;34(8): 978-85.

34. Brand $\mathrm{AH}$, Perrimon N. Targeted gene expression as a means of altering cell fates and generating dominant phenotypes. Development. 1993:118:401-5.

35. Fogarty P, Campbell SD, Abu-Shumays R, Phalle BS, Yu KR, Uy GL, et al. The Drosophila grapes gene is related to checkpoint gene chk1/rad27 and is required for late syncytial division fidelity. Curr Biol 1997; 7(6): 418-426.

36. Lu WJ, Chapo J, Roig I, Abrams JM. Meiotic recombination provokes functional activation of the p53 regulatory network. Science. 2010;328:1278-81.

37. Wylie A, Lu WJ, D'Brot A, Buszcak M. Abrams JM. p53 activity is selectively licensed in the Drosophila stem cell compartment. eLIFE. 2014;3:e01530.

38. Bayer FE, Zimmermann M, Fischer P, Gromoll C, Preiss A. Nagel AC. p53 and cyclin $G$ cooperate in mediating genome stability in somatic cells of Drosophila. Sci Rep. 2017;7(1):17890. https://doi.org/10.1038/s41598-017-17973-z

39. Kugler SJ, Nagel AC. putzig is required for cell proliferation and regulates notch activity in Drosophila. Mol Biol Cell. 2007;18:3733-40.

40. Huang J, Zhou W, Dong W, Watson AM, Hong Y. From the cover: directed, efficient, and versatile modifications of the Drosophila genome by genomic engineering. Proc Natl Acad Sci U S A. 2009;106:8284-9.

41. Rubin GM, Spradling AC. Genetic transformation of Drosophila with transposable element vectors. Science. 1992;218:348-53.

42. Tautz D, Pfeifle C. A non-radioactive in situ hybridization method for the localization of specific RNAs in Drosophila embryos reveals translational control of the segmentation gene hunchback. Chromosoma. 1989:98:81-5.

43. Praxenthaler H, Nagel AC, Schulz A, Zimmermann M, Meier M, Schmid A, et al. Hairless-binding deficient Suppressor of Hairless alleles reveal Su(H) protein levels are dependent on complex formation with hairless. PLOS Genet. 2017;13:e1006774. https://doi.org/10.1371/journal.pgen.1006774.

44. Pfaffl MW, Horgan GW, Dempfle L. Relative expression software tool (REST) for a group-wise comparison and statistical analysis of relative expression results in real-time PCR. Nucl Acids Res. 2002;30:e36.

45. Hu Y, Sopko R, Foos F, Kelley C, Flockhart I, Ammeux N, et al. FlyPrimerBank: an online database for Drosophila melanogaster gene expression analysis and knock-down evaluation of RNAi reagents. G3 (Bethesda). 2013;3:1607-16. 
46. Neufeld TP, Edgar BA. Connections between growth and the cell cycle. Curr Opin Cell Biol. 1998;10:784-90.

47. Bischof J, Björklund M, Furger E, Schertel C, Taipale J, Basler K. A versatile platform for creating a comprehensive UAS-ORFeome library in Drosophila. Development. 2013;140(11):2434-42.

48. Bakhrat A, Pritchett T, Peretz G, McCall K, Abdu U. Drosophila Chk2 and p53 proteins induce stage-specific cell death independently during oogenesis. Apoptosis. 2010;15:1425-34.

49. Rørth P. Gal4 in the Drosophila female germline. Mech Dev. 1998;78:113-8.

50. Zimmermann M, Kugler SJ, Schulz A, Nagel AC. Loss of putzig activity results in apoptosis during wing imaginal development in Drosophila. PLoS One. 2015;10(4):e0124652.

- fast, convenient online submission

- thorough peer review by experienced researchers in your field

- rapid publication on acceptance

- support for research data, including large and complex data types

- gold Open Access which fosters wider collaboration and increased citations

- maximum visibility for your research: over $100 \mathrm{M}$ website views per year

At BMC, research is always in progress. 